\title{
On a Nonlocal Multipoint and Integral Boundary Value Problem of Nonlinear Fractional Integrodifferential Equations
}

\author{
Lahcen Ibnelazyz $\mathbb{D}^{\mathbb{D}}$, Karim Guida $\mathbb{D}^{\mathbb{D}}$, Said Melliani, and Khalid Hilal
}

Sultan Moulay Slimane University, BP 523, 23000 Beni Mellal, Morocco

Correspondence should be addressed to Lahcen Ibnelazyz; ibnelazyzlahcen@gmail.com

Received 12 September 2020; Revised 29 September 2020; Accepted 1 October 2020; Published 28 October 2020

Academic Editor: Maria Alessandra Ragusa

Copyright (c) 2020 Lahcen Ibnelazyz et al. This is an open access article distributed under the Creative Commons Attribution License, which permits unrestricted use, distribution, and reproduction in any medium, provided the original work is properly cited.

The aim of this paper is to give the existence as well as the uniqueness results for a multipoint nonlocal integral boundary value problem of nonlinear sequential fractional integrodifferential equations. First of all, we give some preliminaries and notations that are necessary for the understanding of the manuscript; second of all, we show the existence and uniqueness of the solution by means of the fixed point theory, namely, Banach's contraction principle and Krasnoselskii's fixed point theorem. Last, but not least, we give two examples to illustrate the results.

\section{Introduction}

In the last few years, fractional differential equations have gained much attention among mathematicians because of the rapid growth and for their applicability in several fields, such as physics, biology, economics, control theory, and engineering; for more details about the theory of fractional differential equations and their applications, we recommend the following articles [1-15] and the references therein.

Furthermore, fractional differential equations with multipoint boundary conditions have provoked a great deal of attention by many authors; a lot of works have been published on this topic; for more details, we give the following references [16-22].

In a recent paper [23], the existence of solutions for a four-point nonlocal boundary value problem of nonlinear integrodifferential equations of fractional order was proven. In [24], the authors discussed the existence of solutions for fractional differential equations with multipoint boundary conditions. The existence and uniqueness of solutions for multiterm nonlinear fractional integrodifferential equations have been studied in [25]. The existence results for sequential fractional integrodifferential equations with nonlocal multipoint and strip conditions were established in [26], and finally, in [27], the authors studied the existence of solutions for nonlinear fractional integrodifferential equations.

Motivated by all these works, and by the fact that there are no papers dealing with nonlinear fractional integrodifferential equations with multipoint and integral boundary value conditions, in this work, we consider the existence and uniqueness of solutions for the following problem:

$$
\left\{\begin{array}{l}
{ }^{c} D^{\beta}\left({ }^{c} D^{\alpha}\right) x(t)=f(t, x(t), \phi x(t), \varphi x(t)), \quad t \in[0,1], \\
x(0)=\sum_{i=1}^{n} a_{i} x\left(t_{i}\right), x^{\prime}(0)=0, \mu_{1} x(1)+\mu_{2} x^{\prime}(1)=\sum_{j=1}^{m} b_{j} \int_{c_{j}}^{d_{j}} x(s) d s,
\end{array}\right.
$$

where $0<\alpha<1,1<\beta \leq 2$, with $\alpha+\beta-2 \geq 0, \mu_{1}, \mu_{2}, a_{i}, b_{j} \in \mathbb{R}$; $0<t_{i}<c_{j}<d_{j}<1, i=1,2, \cdots, n, j=1,2, \cdots, m,{ }^{c} D^{\alpha},{ }^{c} D^{\beta}$ are the Caputo fractional derivatives, and $f:[0,1] \times \mathbb{R}^{3} \rightarrow \mathbb{R}$ is a continuous function and $\phi x(t)=\int_{0}^{t} \lambda(t, s) x(s) d s, \varphi x(t)=\int_{0}^{t} \delta$ 
$(t, s) x(s) d s$, where $\lambda, \delta:[0,1] \times[0,1] \rightarrow[0,+\infty)$, with $\lambda_{0}=$ $\sup _{t \in[0,1]}\left|\int_{0}^{t} \lambda(t, s) d s\right|<\infty, \delta_{0}=\sup _{t \in[0,1]}\left|\int_{0}^{t} \delta(t, s) d s\right|<\infty$.

This paper is organized as follows: in the second section, we give some preliminaries and notations that will be useful throughout the work; after that, in the third section, we establish the main results by using the fixed point theory; and in the last section, we give some examples to illustrate the results.

\section{Preliminaries and Notations}

Throughout this section, we present some notations, definitions, and lemmas which will be used for the rest of the paper.

Definition 1 (see [5]). The fractional integral of order $\alpha>0$ with the lower limit zero for a function $f$ can be defined as

$$
I^{\alpha} f(t)=\frac{1}{\Gamma(\alpha)} \int_{0}^{t}(t-s)^{\alpha-1} f(s) d s .
$$

Definition 2 (see [5]). The Caputo derivative of order $\alpha>0$ with the lower limit zero for a function $f$ can be defined as

$$
{ }^{c} D^{\alpha} f=\frac{1}{\Gamma(n-\alpha)} \int_{0}^{t}(t-s)^{n-\alpha-1} f^{(n)}(s) d s .
$$

where $n \in \mathbb{N}, 0 \leq n-1<\alpha<n, t>0$.

Theorem 3 (see [28]). Let $M$ be a bounded, closed, convex, and nonempty subset of a Banach space $X$. Let $A$ and $B$ be two operators such that

(i) $A x+B y \in M$ whenever $x, y \in M$

(ii) A is compact and continuous

(iii) $B$ is a contraction mapping

Then, there exists $z \in M$ such that $z=A z+B z$.

Lemma 4 (see [5]). Let $\alpha, \beta \geq 0$, then the following relation holds:

$$
I^{\alpha} t^{\beta}=\frac{\Gamma(\beta+1)}{\Gamma(\alpha+\beta+1)} t^{\alpha+\beta}
$$

Lemma 5 (see [5]). Let $n \in \mathbb{N}$ and $n-1<\alpha<n$. If $f$ is a continuous function, then we have

$$
I^{\alpha c} D^{\alpha} f(t)=f(t)+a_{0}+a_{1} t+a_{2} t^{2}+\cdots+a_{n-1} t^{n-1} .
$$

Lemma 6. Let $h \in C([0,1], \mathbb{R})$. Then, the unique solution of the boundary value problem

$$
\left\{\begin{array}{l}
{ }^{c} D^{\beta}\left({ }^{c} D^{\alpha}\right) x(t)=h(t), \quad t \in[0,1], \\
x(0)=\sum_{i=1}^{n} a_{i} x\left(t_{i}\right), x^{\prime}(0)=0, \mu_{1} x(1)+\mu_{2} x^{\prime}(1)=\sum_{j=1}^{m} b_{j} \int_{c_{j}}^{d_{j}} x(s) d s,
\end{array}\right.
$$

is given by

$$
\begin{aligned}
x(t)= & \frac{1}{\Gamma(\alpha+\beta)} \int_{0}^{t}(t-s)^{\alpha+\beta-1} h(s) d s \\
& -\frac{B_{1}(t)}{\Gamma(\alpha+\beta)} \sum_{i=1}^{n} a_{i} \int_{0}^{t_{i}}\left(t_{i}-s\right)^{\alpha+\beta-1} \times h(s) d s \\
& +B_{2}(t)\left[\sum_{j=1}^{m} \frac{b_{j}}{\Gamma(\alpha+\beta)} \int_{c_{j}}^{d_{j}}\left(\int_{0}^{s}(s-u)^{\alpha+\beta-1} h(u) d u\right) d s\right. \\
& -\frac{\mu_{1}}{\Gamma(\alpha+\beta)} \int_{0}^{1}(1-s)^{\alpha+\beta-1} h(s) d s-\frac{\mu_{2}}{\Gamma(\alpha+\beta-1)} \\
& \left.\times \int_{0}^{t}(1-s)^{\alpha+\beta-2} h(s) d s\right],
\end{aligned}
$$

where

$$
\begin{gathered}
\Delta=L_{1} K_{2}-L_{2} K_{1} \neq 0, L_{1}=-\frac{\sum_{i=1}^{n} a_{i} t_{i}^{\alpha+1}}{\Gamma(\alpha+2)}, L_{2}=1-\sum_{j=1}^{n} a_{i}, \\
K_{1}=\frac{\mu_{1}}{\Gamma(\alpha+2)}+\frac{\mu_{2}}{\Gamma(\alpha+1)}-\frac{\sum_{j=1}^{m} b_{j}\left(d_{j}^{\alpha+2}-c_{j}^{\alpha+2}\right)}{\Gamma(\alpha+3)}, \\
K_{2}=\mu_{1}-\sum_{j=1}^{m} b_{j}\left(d_{j}-c_{j}\right), B_{1}(t)=\frac{1}{\Delta}\left(K_{1}-\frac{K_{2} t^{\alpha+1}}{\Gamma(\alpha+2)}\right), \\
B_{2}(t)=\frac{1}{\Delta}\left(L_{1}-\frac{L_{2} t^{\alpha+1}}{\Gamma(\alpha+2)}\right) .
\end{gathered}
$$

Proof. By applying Lemma 5, we obtain

$$
\begin{aligned}
{ }^{c} D^{\alpha} x(t) & =I^{\beta} h(t)+\theta_{0}+\theta_{1} t, \\
x(t) & =I^{\alpha+\beta} h(t)+I^{\alpha} \theta_{0}+I^{\alpha} \theta_{1} t+\theta_{2},
\end{aligned}
$$

where $\theta_{0}, \theta_{1}, \theta_{2} \in \mathbb{R}$. 
This means that

$$
\begin{aligned}
x(t)= & \frac{1}{\Gamma(\alpha+\beta)} \int_{0}^{t}(t-s)^{\alpha+\beta-1} h(s) d s+\frac{t^{\alpha}}{\Gamma(\alpha+1)} \theta_{0} \\
& +\frac{t^{\alpha+1}}{\Gamma(\alpha+2)} \theta_{1}+\theta_{2}, \\
x^{\prime}(t)= & \frac{1}{\Gamma(\alpha+\beta-1)} \int_{0}^{t}(t-s)^{\alpha+\beta-2} h(s) d s+\frac{t^{\alpha-1}}{\Gamma(\alpha)} \theta_{0} \\
& +\frac{t^{\alpha}}{\Gamma(\alpha+1)} \theta_{1},
\end{aligned}
$$

and by using the condition $x^{\prime}(0)=0$, we get $\theta_{0}=0$.

As a result of the condition $x(0)=\sum_{i=1}^{n} a_{i} x\left(t_{i}\right)$, we find that $L_{2} \theta_{2}+L_{1} \theta_{1}=H_{1}$, where

$$
H_{1}=\sum_{i=1}^{n} \frac{a_{i}}{\Gamma(\alpha+\beta)} \int_{0}^{t_{i}}\left(t_{i}-s\right)^{\alpha+\beta-1} h(s) d s .
$$

Now, we use the condition $\mu_{1} x(1)+\mu_{2} x^{\prime}(1)=\sum_{j=1}^{m} b_{j} \int_{c_{j}}^{d_{j}}$ $x(s) d s$, to obtain $K_{2} \theta_{2}+K_{1} \theta_{1}=H_{2}$, where

$$
\begin{aligned}
H_{2}= & \frac{-\mu_{1}}{\Gamma(\alpha+\beta)} \int_{0}^{1}(1-s)^{\alpha+\beta-1} h(s) d s \\
& -\frac{\mu_{2}}{\Gamma(\alpha+\beta-1)} \int_{0}^{1}(1-s)^{\alpha+\beta-2} \times h(s) d s \\
& +\sum_{j=1}^{m} \frac{b_{j}}{\Gamma(\alpha+\beta)} \int_{c_{j}}^{d_{i}}\left(\int_{0}^{s}(s-u)^{\alpha+\beta-1} h(u) d u\right) d s .
\end{aligned}
$$

Finally, we have

$$
\theta_{1}=\frac{K_{2} H_{1}-L_{2} H_{2}}{\Delta}, \theta_{2}=\frac{L_{1} H_{2}-K_{1} H_{1}}{\Delta} .
$$

By substituting the value of $\theta_{0}, \theta_{1}$, and $\theta_{2}$, we get the following:

$$
\begin{aligned}
x(t)= & \frac{1}{\Gamma(\alpha+\beta)} \int_{0}^{t}(t-s)^{\alpha+\beta-1} h(s) d s \\
& -\frac{B_{1}(t)}{\Gamma(\alpha+\beta)} \sum_{i=1}^{n} a_{i} \int_{0}^{t_{i}}\left(t_{i}-s\right)^{\alpha+\beta-1} \times h(s) d s \\
& +B_{2}(t)\left[\sum_{j=1}^{m} \frac{b_{j}}{\Gamma(\alpha+\beta)} \int_{c_{j}}^{d_{j}}\left(\int_{0}^{s}(s-u)^{\alpha+\beta-1} h(u) d u\right) d s\right. \\
& -\frac{\mu_{1}}{\Gamma(\alpha+\beta)} \int_{0}^{1}(1-s)^{\alpha+\beta-1} h(s) d s-\frac{\mu_{2}}{\Gamma(\alpha+\beta-1)} \\
& \left.\times \int_{0}^{1}(1-s)^{\alpha+\beta-2} h(s) d s\right] .
\end{aligned}
$$

Conversely, by direct computations, we obtain the desired result.

\section{Main Results}

Let $X$ be the Banach space of all continuous functions from $[0,1] \rightarrow \mathbb{R}$ endowed with norm $\|x\|=\sup \{|x(t)|: t \in[0,1]\}$.

Theorem 7. Suppose that $f:[0,1] \times \mathbb{R}^{3} \rightarrow \mathbb{R}$ is a continuous function satisfying

$\left(H_{1}\right)$ for all $t \in[0,1]$ and $x_{1}, x_{2}, x_{3}, y_{1}, y_{2}, y_{3} \in \mathbb{R}$, we have $\left|f\left(t, x_{1}, x_{2}, x_{3}\right)-f\left(t, y_{1}, y_{2}, y_{3}\right)\right| \leq \sigma(t)\left(\left|x_{1}-y_{1}\right|+\left|x_{2}-y_{2}\right|+\right.$ $\left.\left|x_{3}-y_{3}\right|\right)$ with $\sigma(t) \in L^{1}([0,1] ;[0, \infty))$.

Then, there exists a unique solution for problem (1) under the following condition: $r_{1}<1$, where

$$
\begin{aligned}
r_{1}= & \left(1+\lambda_{0}+\delta_{0}\right) \sigma^{*} \\
& \cdot\left(\frac{1+B_{1} \sum_{i=1}^{n}\left|a_{i}\right|+B_{2} \sum_{j=1}^{m}\left|b_{j}\right|\left(d_{j}-c_{j}\right)+B_{2}\left|\mu_{1}\right|}{\Gamma(\alpha+\beta)}+\frac{B_{2}\left|\mu_{2}\right|}{\Gamma(\alpha+\beta-1)}\right),
\end{aligned}
$$

with $B_{1}=1 /|\Delta|\left(\left|K_{1}\right|+\left|K_{2}\right| / \Gamma(\alpha+\beta)\right), B_{2}=1 /|\Delta|\left(\left|L_{1}\right|+\left|L_{2}\right| /\right.$ $\Gamma(\alpha+2))$, and $\sigma^{*}=\int_{0}^{1} \sigma(t) d t$.

Proof. Define $P: X \rightarrow X$ by

$$
\begin{aligned}
P x(t)= & \frac{1}{\Gamma(\alpha+\beta)} \int_{0}^{t}(t-s)^{\alpha+\beta-1} f(s, x(s), \phi x(s), \varphi x(s)) d s \\
& -\frac{B_{1}(t)}{\Gamma(\alpha+\beta)} \times \sum_{i=1}^{n} a_{i} \int_{0}^{t_{i}}\left(t_{i}-s\right)^{\alpha+\beta-1} f(s, x(s), \phi x(s), \varphi x(s)) d s \\
& +B_{2}(t) \times\left[\sum _ { j = 1 } ^ { m } \frac { b _ { j } } { \Gamma ( \alpha + \beta ) } \int _ { c _ { j } } ^ { d _ { j } } \left(\int_{0}^{s}(s-u)^{\alpha+\beta-1} f\right.\right. \\
& \cdot(u, x(u), \phi x(u), \varphi x(u)) \times d u) d s \\
& -\frac{\mu_{1}}{\Gamma(\alpha+\beta)} \int_{0}^{1}(1-s)^{\alpha+\beta-1} f(s, x(s), \phi x(s), \varphi x(s)) d s \\
& \left.-\frac{\mu_{2}}{\Gamma(\alpha+\beta-1)} \int_{0}^{1}(1-s)^{\alpha+\beta-2} f(s, x(s), \phi x(s), \varphi x(s)) d s\right] .
\end{aligned}
$$

Setting $\sup _{0 \leq t \leq 1}|f(t, 0,0,0)|=F$.

We consider the following set $B_{r}=\{x \in X:\|x\| \leq r\}$, where $r \geq r_{2} /\left(1-r_{1}\right)$, with

$$
\begin{aligned}
r_{2}= & \frac{F}{\Gamma(\alpha+\beta)}+\frac{B_{1}}{\Gamma(\alpha+\beta)} \sum_{i=1}^{n}\left|a_{i}\right| F+B_{2} \sum_{j=1}^{m} \frac{\left|b_{j}\right| F\left(d_{j}-c_{j}\right)}{\Gamma(\alpha+\beta)} \\
& +\frac{B_{2}\left|\mu_{1}\right| F}{\Gamma(\alpha+\beta)}+\frac{B_{2}\left|\mu_{2}\right| F}{\Gamma(\alpha+\beta-1)} .
\end{aligned}
$$


For each $t \in[0,1]$ and $x \in B_{r}$, we have

$$
\begin{aligned}
& |P x(t)| \leq \frac{1}{\Gamma(\alpha+\beta)} \int_{0}^{t}(t-s)^{\alpha+\beta-1}|f(s, x(s), \phi x(s), \varphi x(s))| d s \\
& +\frac{\left|B_{1}(t)\right|}{\Gamma(\alpha+\beta)} \times \sum_{i=1}^{n}\left|a_{i}\right| \int_{0}^{t_{i}}\left(t_{i}-s\right)^{\alpha+\beta-1} \\
& \cdot|f(s, x(s), \phi x(s), \varphi x(s))| d s+\left|B_{2}(t)\right| \\
& \cdot\left[\sum _ { j = 1 } ^ { m } \frac { | b _ { j } | } { \Gamma ( \alpha + \beta ) } \int _ { c _ { j } } ^ { d _ { j } } \left(\int_{0}^{s}(s-u)^{\alpha+\beta-1}\right.\right. \\
& \times|f(u, x(u), \phi x(u), \varphi x(u))| d u) d s+\frac{\left|\mu_{1}\right|}{\Gamma(\alpha+\beta)} \int_{0}^{1} \\
& \cdot(1-s)^{\alpha+\beta-1} \times|f(s, x(s), \phi x(s), \varphi x(s))| d s+\frac{\left|\mu_{2}\right|}{\Gamma(\alpha+\beta-1)} \\
& \left.\cdot \int_{0}^{1}(1-s)^{\alpha+\beta-2} \times|f(s, x(s), \phi x(s), \varphi x(s))| d s\right] \\
& \leq \frac{1}{\Gamma(\alpha+\beta)} \int_{0}^{t}(t-s)^{\alpha+\beta-1}(\mid f(s, x(s), \phi x(s), \varphi x(s)) \\
& -f(s, 0,0,0)|+| f(s, 0,0,0) \mid) d s+\frac{\left|B_{1}(t)\right|}{\Gamma(\alpha+\beta)} \sum_{i=1}^{n}\left|a_{i}\right| \int_{0}^{t_{i}} \\
& \cdot\left(t_{i}-s\right)^{\alpha+\beta-1} \times(|f(s, x(s), \phi x(s), \varphi x(s))-f(s, 0,0,0)| \\
& +|f(s, 0,0,0)|) d s+\left|B_{2}(t)\right|\left[\sum_{j=1}^{m} \frac{\left|b_{j}\right|}{\Gamma(\alpha+\beta)} \int_{c_{j}}^{d_{j}}\right. \\
& \left(\int_{0}^{s}(s-u)^{\alpha+\beta-1} \times(\mid f(u, x(u), \phi x(u), \varphi x(u))\right. \\
& -f(u, 0,0,0)|+| f(u, 0,0,0) \mid) d u) d s+\frac{\left|\mu_{1}\right|}{\Gamma(\alpha+\beta)} \int_{0}^{1} \\
& \cdot(1-s)^{\alpha+\beta-1}(|f(s, x(s), \phi x(s), \varphi x(s))-f(s, 0,0,0)| \\
& +|f(s, 0,0,0)|) d s+\frac{\left|\mu_{2}\right|}{\Gamma(\alpha+\beta-1)} \int_{0}^{1}(1-s)^{\alpha+\beta-2} \\
& \times(|f(s, x(s), \phi x(s), \varphi x(s))-f(s, 0,0,0)|+|f(s, 0,0,0)|) d s]
\end{aligned}
$$$$
\leq \frac{1}{\Gamma(\alpha+\beta)} \int_{0}^{t}(t-s)^{\alpha+\beta-1}(\sigma(s)(|x(s)|+|\phi x(s)|+|\varphi x(s)|)+F) d s
$$$$
+\frac{B_{1}}{\Gamma(\alpha+\beta)} \sum_{i=1}^{n}\left|a_{i}\right| \int_{0}^{t_{i}}\left(t_{i}-s\right)^{\alpha+\beta-1}(\sigma(s)(|x(s)|+|\phi x(s)|
$$$$
+|\varphi x(s)|)+F) d s+B_{2}\left[\sum_{j=1}^{m} \frac{\left|b_{j}\right|}{\Gamma(\alpha+\beta)} \int_{c_{j}}^{d_{j}} \times \int_{0}^{s}(s-u)^{\alpha+\beta-1}\right.
$$$$
\cdot(\sigma(u)(|x(u)|+|\phi x(u)|+|\varphi x(u)|)+F) d u d s+\frac{\left|\mu_{1}\right|}{\Gamma(\alpha+\beta)} \int_{0}^{1}
$$$$
\cdot(1-s)^{\alpha+\beta-1}(\sigma(s)(|x(s)|+|\phi x(s)|+|\varphi x(s)|)+F) d s
$$$$
+\frac{\left|\mu_{2}\right|}{\Gamma(\alpha+\beta-1)} \int_{0}^{1}(1-s)^{\alpha+\beta-2}(\sigma(s)(|x(s)|+|\phi x(s)|
$$$$
+|\varphi x(s)|)+F) d s] \leq \frac{\left(1+\lambda_{0}+\delta_{0}\right)\|x\|}{\Gamma(\alpha+\beta)} \int_{0}^{1} \sigma(s) d s+\frac{F}{\Gamma(\alpha+\beta)} \int_{0}^{t}
$$$$
\cdot(t-s)^{\alpha+\beta-1} d s+\frac{B_{1}}{\Gamma(\alpha+\beta)} \sum_{i=1}^{n}\left|a_{i}\right|\left(1+\lambda_{0}+\delta_{0}\right)\|x\| \int_{0}^{1} \sigma(s) d s
$$$$
+\frac{B_{1}}{\Gamma(\alpha+\beta)} \sum_{i=1}^{n}\left|a_{i}\right| F \times \int_{0}^{t_{i}}\left(t_{i}-s\right)^{\alpha+\beta-1} d s+B_{2} \sum_{j=1}^{m} \frac{\left|b_{j}\right|}{\Gamma(\alpha+\beta)} \int_{c_{j}}^{d_{j}}
$$$$
\left(\left(1+\lambda_{0}+\delta_{0}\right)\|x\| \times \int_{0}^{1} \sigma(u) d u\right)+B_{2} \sum_{j=1}^{m} \frac{\left|b_{j}\right| F\left(d_{j}-c_{j}\right)}{\Gamma(\alpha+\beta)}
$$

$$
\begin{aligned}
& +\frac{B_{2}\left|\mu_{1}\right|}{\Gamma(\alpha+\beta)}\left(1+\lambda_{0}+\delta_{0}\right)\|x\| \times \int_{0}^{1} \sigma(s) d s+\frac{B_{2}\left|\mu_{1}\right| F}{\Gamma(\alpha+\beta)} \int_{0}^{1} \\
& \times(1-s)^{\alpha+\beta-1} d s+\frac{B_{2}\left|\mu_{2}\right|}{\Gamma(\alpha+\beta-1)}\left(1+\lambda_{0}+\delta_{0}\right)\|x\| \\
& \times \int_{0}^{1} \sigma(s) d s+\frac{B_{2}\left|\mu_{2}\right| F}{\Gamma(\alpha+\beta-1)} \int_{0}^{1}(1-s)^{\alpha+\beta-2} d s \\
& \leq \frac{\left(1+\lambda_{0}+\delta_{0}\right)\|x\|}{\Gamma(\alpha+\beta)} \sigma^{*}+\frac{F}{\Gamma(\alpha+\beta)}+\frac{B_{1}}{\Gamma(\alpha+\beta)} \sum_{i=1}^{n}\left|a_{i}\right| \\
& \times\left(1+\lambda_{0}+\delta_{0}\right)\|x\| \sigma^{*}+\frac{B_{1}}{\Gamma(\alpha+\beta)} \sum_{i=1}^{n}\left|a_{i}\right| F \\
& +B_{2} \sum_{j=1}^{m} \frac{\left|b_{j}\right|\left(d_{j}-c_{j}\right)}{\Gamma(\alpha+\beta)}\left(1+\lambda_{0}+\delta_{0}\right)\|x\| \sigma^{*} \\
& +B_{2} \sum_{j=1}^{m} \frac{\left|b_{j}\right| F\left(d_{j}-c_{j}\right)}{\Gamma(\alpha+\beta)}+\frac{B_{2}\left|\mu_{1}\right|}{\Gamma(\alpha+\beta)}\left(1+\lambda_{0}+\delta_{0}\right)\|x\| \sigma^{*} \\
& +\frac{B_{2}\left|\mu_{1}\right| F}{\Gamma(\alpha+\beta)}+\frac{B_{2}\left|\mu_{2}\right|}{\Gamma(\alpha+\beta-1)}\left(1+\lambda_{0}+\delta_{0}\right) \sigma^{*}\|x\|+\frac{B_{2}\left|\mu_{2}\right| F}{\Gamma(\alpha+\beta-1)} \\
& \leq\left[\frac{\left(1+\lambda_{0}+\delta_{0}\right) \sigma^{*}}{\Gamma(\alpha+\beta)}+\frac{B_{1}}{\Gamma(\alpha+\beta)} \sum_{i=1}^{n}\left|a_{i}\right|\left(1+\lambda_{0}+\delta_{0}\right) \sigma^{*}+B_{2}\right. \\
& \times \sum_{j=1}^{m} \frac{\left|b_{j}\right|\left(d_{j}-c_{j}\right)}{\Gamma(\alpha+\beta)}\left(1+\lambda_{0}+\delta_{0}\right) \sigma^{*}+\frac{B_{2}\left|\mu_{1}\right|}{\Gamma(\alpha+\beta)} \\
& \left.\times\left(1+\lambda_{0}+\delta_{0}\right) \sigma^{*}+\frac{B_{2}\left|\mu_{2}\right|}{\Gamma(\alpha+\beta-1)}\left(1+\lambda_{0}+\delta_{0}\right) \sigma^{*}\right]\|x\| \\
& +\frac{F}{\Gamma(\alpha+\beta)}+\frac{B_{1}}{\Gamma(\alpha+\beta)} \times \sum_{i=1}^{n}\left|a_{i}\right| F+B_{2} \sum_{j=1}^{m} \frac{\left|b_{j}\right| F\left(d_{j}-c_{j}\right)}{\Gamma(\alpha+\beta)} \\
& +\frac{B_{2}\left|\mu_{1}\right| F}{\Gamma(\alpha+\beta)}+\frac{B_{2}\left|\mu_{2}\right| F}{\Gamma(\alpha+\beta-1)} \text {. }
\end{aligned}
$$

This means that $\|P x\| \leq r$.

Therefore, $P B_{r} \subseteq B_{r}$.

Next, we prove that $P$ is a contraction mapping. For $x, y \in B_{r}$, we have

$$
\begin{aligned}
\mid P x(t) & -P y(t) \mid \\
\leq & \frac{1}{\Gamma(\alpha+\beta)} \int_{0}^{t}(t-s)^{\alpha+\beta-1}(\mid f(s, x(s), \phi x(s), \varphi x(s)) \\
& -f(s, y(s), \phi y(s), \varphi y(s)) \mid) d s+\frac{B_{1} \sum_{i=1}^{n}\left|a_{i}\right|}{\Gamma(\alpha+\beta)} \\
& \times \int_{0}^{t_{i}}\left(t_{i}-s\right)^{\alpha+\beta-1}(\mid f(s, x(s), \phi x(s), \varphi x(s)) \\
& -f(s, y(s), \phi y(s), \varphi y(s)) \mid) d s \\
& +B_{2}\left[\sum_{j=1}^{m} \frac{\left|b_{j}\right|}{\Gamma(\alpha+\beta)} \times \int_{c_{j}}^{d_{j}}\right. \\
& \times\left(\int_{0}^{s}(s-u)^{\alpha+\beta-1}(\mid f(s, x(u), \phi x(u), \varphi x(u))\right. \\
& -f(s, y(u), \phi y(u), \varphi y(u)) \mid) d u) d s
\end{aligned}
$$




$$
\begin{aligned}
& +\frac{\left|\mu_{1}\right|}{\Gamma(\alpha+\beta)} \times \int_{0}^{1}(1-s)^{\alpha+\beta-1}(\mid f(s, x(s), \phi x(s), \varphi x(s)) \\
& -f(s, y(s), \phi y(s), \varphi y(s)) \mid) d s+\frac{\left|\mu_{2}\right|}{\Gamma(\alpha+\beta-1)} \\
& \left.\times \int_{0}^{1}(1-s)^{\alpha+\beta-2}(|f(s, x(s), \phi x(s), \varphi x(s))-f(s, y(s), \phi y(s), \varphi y(s))|) d s\right] \\
& \leq \frac{1}{\Gamma(\alpha+\beta)} \int_{0}^{t}(t-s)^{\alpha+\beta-1} \sigma(s)(|x(s)-y(s)|+\mid \phi x(s) \\
& -\phi y(s)|+| \varphi x(s)-\varphi y(s) \mid) d s+\frac{B_{1}}{\Gamma(\alpha+\beta)} \sum_{i=1}^{n}\left|a_{i}\right| \int_{0}^{t_{i}} \\
& \times\left(t_{i}-s\right)^{\alpha+\beta-1} \sigma(s) \times(|x(s)-y(s)|+\mid \phi x(s) \\
& -\phi y(s)|+| \varphi x(s)-\varphi y(s) \mid) d s+B_{2}\left[\sum_{j=1}^{m} \frac{\left|b_{j}\right|}{\Gamma(\alpha+\beta)} \int_{c_{j}}^{d_{j}}\right. \\
& \times\left(\int_{0}^{s}(s-u)^{\alpha+\beta-1} \sigma(u) \times(|x(u)-y(u)|+\mid \phi x(u)\right. \\
& -\phi y(u)|+| \varphi x(u)-\varphi y(u) \mid) d u) d s \\
& +\frac{\left|\mu_{1}\right|}{\Gamma(\alpha+\beta)} \int_{0}^{1}(1-s)^{\alpha+\beta-1} \sigma(s)(|x(s)-y(s)|+\mid \phi x(s) \\
& -\phi y(s)|+| \varphi x(s)-\varphi y(s) \mid) d s+\frac{\left|\mu_{2}\right|}{\Gamma(\alpha+\beta-1)} \\
& \times \int_{0}^{1}(1-s)^{\alpha+\beta-2} \sigma(s)(|x(s)-y(s)|+\mid \phi x(s) \\
& \times-\phi y(s)|+| \varphi x(s)-\varphi y(s) \mid) d s] \\
& \leq \frac{\left(1+\lambda_{0}+\delta_{0}\right)\|x-y\|}{\Gamma(\alpha+\beta)} \int_{0}^{1} \sigma(s) d s \\
& +\frac{B_{1}}{\Gamma(\alpha+\beta)} \sum_{i=1}^{n}\left|a_{i}\right|\left(1+\lambda_{0}+\delta_{0}\right)\|x-y\| \int_{0}^{1} \sigma(s) d s \\
& +B_{2} \sum_{j=1}^{m} \frac{\left|b_{j}\right|}{\Gamma(\alpha+\beta)} \int_{c_{j}}^{d_{j}}\left(\left(1+\lambda_{0}+\delta_{0}\right)\|x-y\| \int_{0}^{1} \sigma(u) d u\right) d s \\
& +\frac{B_{2}\left|\mu_{1}\right|}{\Gamma(\alpha+\beta)}\left(1+\lambda_{0}+\delta_{0}\right)\|x-y\| \int_{0}^{1} \sigma(s) d s \\
& +\frac{B_{2}\left|\mu_{2}\right|}{\Gamma(\alpha+\beta-1)}\left(1+\lambda_{0}+\delta_{0}\right)\|x-y\| \int_{0}^{1} \sigma(s) d s \\
& \leq \frac{\left(1+\lambda_{0}+\delta_{0}\right)\|x-y\|}{\Gamma(\alpha+\beta)} \sigma^{*}+\frac{B_{1}}{\Gamma(\alpha+\beta)} \sum_{i=1}^{n}\left|a_{i}\right|\left(1+\lambda_{0}+\delta_{0}\right)\|x-y\| \sigma^{*} \\
& +B_{2} \sum_{j=1}^{m} \frac{\left|b_{j}\right|\left(d_{j}-c_{j}\right)}{\Gamma(\alpha+\beta)}\left(1+\lambda_{0}+\delta_{0}\right) \sigma^{*}\|x-y\| \\
& +\frac{B_{2}\left|\mu_{1}\right|}{\Gamma(\alpha+\beta)}\left(1+\lambda_{0}+\delta_{0}\right) \sigma^{*}\|x-y\| \\
& +\frac{B_{2}\left|\mu_{2}\right|}{\Gamma(\alpha+\beta-1)}\left(1+\lambda_{0}+\delta_{0}\right) \sigma^{*}\|x-y\| \leq\left(1+\lambda_{0}+\delta_{0}\right) \sigma^{*} \\
& \times\left(\frac{1+B_{1} \sum_{i=1}^{n}\left|a_{i}\right|+B_{2} \sum_{j=1}^{m}\left|b_{j}\right|\left(d_{j}-c_{j}\right)+B_{2}\left|\mu_{1}\right|}{\Gamma(\alpha+\beta)}+\frac{B_{2}\left|\mu_{2}\right|}{\Gamma(\alpha+\beta-1)}\right)\|x-y\| .
\end{aligned}
$$

Since $r_{1}<1$, then $P$ is a contraction. Therefore, system (1) has a unique solution.

Theorem 8. Assume that $\left(H_{1}\right)$ holds and $f:[0 ; 1] \times \mathbb{R}^{3} \rightarrow \mathbb{R}$ is a continuous function. Furthermore, we suppose

$\left(H_{3}\right) \quad|f(t, x, y, z)| \leq \theta(t), \quad \forall(t, x, y, z) \in[0,1] \times \mathbb{R}^{3}$ with $\theta \in L^{1}\left([0,1] ; \mathbb{R}^{+}\right)$.
Then, problem (1) has at least one solution on $[0,1]$ if $R$ $<1$, where

$$
\begin{aligned}
R= & \left(1+\lambda_{0}+\delta_{0}\right) \sigma^{*} \\
& \cdot\left(\frac{B_{1} \sum_{i=1}^{n}\left|a_{i}\right|+B_{2} \sum_{j=1}^{m}\left|b_{j}\right|\left(d_{j}-c_{j}\right)+B_{2}\left|\mu_{1}\right|}{\Gamma(\alpha+\beta)}+\frac{B_{2}\left|\mu_{2}\right|}{\Gamma(\alpha+\beta-1)}\right) .
\end{aligned}
$$

Proof. We now consider the closed ball $B_{r^{\prime}}=\{x \in X:\|x\| \leq$ $\left.r^{\prime}\right\}$ with fixed radius $r^{\prime}$ :

$$
\begin{aligned}
r^{\prime} \geq & \frac{\|\theta\|_{L^{1}}}{\Gamma(\alpha+\beta+1)}+\frac{B_{1}}{\Gamma(\alpha+\beta+1)} \sum_{i=1}^{n}\left|a_{i}\right| t_{i}^{\alpha+\beta}\|\theta\|_{L^{1}} \\
& +B_{2}\left[\sum_{j=1}^{m} \frac{\left|b_{j}\right|\|\theta\|_{L^{1}}\left(d_{j}-c_{j}\right)}{\Gamma(\alpha+\beta+1)}+\frac{\left|\mu_{1}\right|\|\theta\|_{L^{1}}}{\Gamma(\alpha+\beta+1)}+\frac{\left|\mu_{2}\right|\|\theta\|_{L^{1}}}{\Gamma(\alpha+\beta)}\right] .
\end{aligned}
$$

We define the operators $P_{1}$ and $P_{2}$ on $B_{r^{\prime}}$ as

$$
\begin{aligned}
P_{1} x(t)= & \frac{1}{\Gamma(\alpha+\beta)} \int_{0}^{t}(t-s)^{\alpha+\beta-1} f(s, x(s), \phi x(s), \varphi x(s)) d s \\
P_{2} x(t)= & -\frac{B_{1}(t) \sum_{i=1}^{n} a_{i}}{\Gamma(\alpha+\beta)} \int_{0}^{t_{i}}\left(t_{i}-s\right)^{\alpha+\beta-1} f(s, x(s), \phi x(s), \varphi x(s)) d s \\
& +B_{2}(t) \times\left[\sum _ { j = 1 } ^ { m } \frac { b _ { j } } { \Gamma ( \alpha + \beta ) } \int _ { c _ { j } } ^ { d _ { j } } \left(\int_{0}^{s}(s-u)^{\alpha+\beta-1} f\right.\right. \\
& \cdot(u, x(u), \phi x(u), \varphi x(u)) \times d u) d s-\frac{\mu_{1}}{\Gamma(\alpha+\beta)} \int_{0}^{1} \\
& \cdot(1-s)^{\alpha+\beta-1} f(s, x(s), \phi x(s), \varphi x(s)) d s-\frac{\mu_{2}}{\Gamma(\alpha+\beta-1)} \int_{0}^{1} \\
& \left.\cdot(1-s)^{\alpha+\beta-2} f(s, x(s), \phi x(s), \varphi x(s)) d s\right] .
\end{aligned}
$$

For $x, y \in B_{r^{\prime}}$, we have

$$
\left|P_{1} x(t)\right| \leq \frac{\|\theta\|_{L^{1}}}{\Gamma(\alpha+\beta+1)},
$$

$$
\begin{aligned}
\left|P_{2} y(t)\right| \leq & \frac{B_{1}}{\Gamma(\alpha+\beta+1)} \sum_{i=1}^{n}\left|a_{i}\right| t_{i}^{\alpha+\beta}\|\theta\|_{L^{1}} \\
& +B_{2}\left[\sum_{j=1}^{m} \frac{\left|b_{j}\right|\|\theta\|_{L^{1}}\left(d_{j}-c_{j}\right)}{\Gamma(\alpha+\beta+1)}+\frac{\left|\mu_{1}\right|\|\theta\|_{L^{1}}}{\Gamma(\alpha+\beta+1)}\right. \\
& \left.+\frac{\left|\mu_{2}\right|\|\theta\|_{L^{1}}}{\Gamma(\alpha+\beta+1)}\right] .
\end{aligned}
$$

Consequently, 


$$
\begin{aligned}
& \| P_{1} x+P_{2} y \| \\
& \leq \frac{\|\theta\|_{L^{1}}}{\Gamma(\alpha+\beta+1)}+\frac{B_{1}}{\Gamma(\alpha+\beta+1)} \sum_{i=1}^{n}\left|a_{i}\right| t_{i}^{\alpha+\beta}\|\theta\|_{L^{1}}+B_{2} \\
& \quad \times\left[\sum_{j=1}^{m} \frac{\left|b_{j}\right|\|\theta\|_{L^{1}}\left(d_{j}-c_{j}\right)}{\Gamma(\alpha+\beta+1)}+\frac{\left|\mu_{1}\right|\|\theta\|_{L^{1}}}{\Gamma(\alpha+\beta+1)}+\frac{\left|\mu_{2}\right|\|\theta\|_{L^{1}}}{\Gamma(\alpha+\beta)}\right] .
\end{aligned}
$$

Then,

$$
P_{1} x+P_{2} y \in B_{r^{\prime}}
$$
have

Next, we show that $P_{2}$ is a contraction. For $x, y \in B_{r^{\prime}}$, we

$$
\begin{aligned}
& \left\|P_{2} x(t)-P_{2} y(t)\right\| \\
& \leq \frac{\left|B_{1}(t)\right|}{\Gamma(\alpha+\beta)} \sum_{i=1}^{n}\left|a_{i}\right| \int_{i=1}^{t_{i}}\left(t_{i}-s\right)^{\alpha+\beta-1} \times \mid f(s, x(s), \phi x(s), \varphi x(s)) \\
& -f(s, y(s), \phi y(s), \varphi y(s))|d s+| B_{2}(t) \mid \\
& \times\left[\sum_{j=1}^{m} \frac{\left|b_{j}\right|}{\Gamma(\alpha+\beta)} \int_{c_{j}}^{d_{j}} \int_{0}^{s}(s-u)^{\alpha+\beta-1} \times \mid f(s, x(u), \phi x(u), \varphi x(u))\right. \\
& -f(s, y(u), \phi y(u), \varphi y(u)) \mid d u d s+\frac{\left|\mu_{1}\right|}{\Gamma(\alpha+\beta)} \int_{0}^{1}(1-s)^{\alpha+\beta-1} \\
& \times|f(s, x(s), \phi x(s), \varphi x(s))-f(s, y(s), \phi y(s), \varphi y(s))| d s \\
& +\frac{\left|\mu_{2}\right|}{\Gamma(\alpha+\beta-1)} \int_{0}^{1}(1-s)^{\alpha+\beta-2} \mid f(s, x(s), \phi x(s), \varphi x(s)) \\
& -f(s, y(s), \phi y(s), \varphi y(s)) \mid d s] \\
& \leq \frac{B_{1}}{\Gamma(\alpha+\beta)} \sum_{i=1}^{n}\left|a_{i}\right| \int_{0}^{t_{i}}(t-s)^{\alpha+\beta-1} \sigma(s)(|x(s)-y(s)|+\mid \phi x(s) \\
& -\phi y(s)|+| \varphi x(s)-\varphi y(s) \mid) d s+B_{2}\left[\sum_{j=1}^{m} \frac{\left|b_{j}\right|}{\Gamma(\alpha+\beta)}\right. \\
& \times \int_{c_{j}}^{d_{j}} \int_{0}^{s}(s-u)^{\alpha+\beta-1} \sigma(u)(|x(u)-y(u)|+\mid \phi x(u) \\
& -\phi y(u)|+| \varphi x(u)-\varphi y(u) \mid) d u d s+\frac{\left|\mu_{1}\right|}{\Gamma(\alpha+\beta)} \int_{0}^{1} \\
& \times(1-s)^{\alpha+\beta-1} \sigma(s)(|x(s)-y(s)|+|\phi x(s)-\phi y(s)|+\mid \varphi x(s) \\
& -\varphi y(s) \mid) d s+\frac{\left|\mu_{2}\right|}{\Gamma(\alpha+\beta-1)} \int_{0}^{1}(1-s)^{\alpha+\beta-2} \sigma(s) \\
& \times(|x(s)-y(s)|+|\phi x(s)-\phi y(s)|+|\varphi x(s)-\varphi y(s)|) d s] \\
& \leq\left(1+\lambda_{0}+\delta_{0}\right) \sigma^{*}\left(\frac{B_{1} \sum_{i=1}^{n}\left|a_{i}\right|+B_{2} \sum_{j=1}^{m}\left|b_{j}\right|\left(d_{j}-c_{j}\right)+B_{2}\left|\mu_{1}\right|}{\Gamma(\alpha+\beta)}\right. \\
& \left.+\frac{B_{2}\left|\mu_{2}\right|}{\Gamma(\alpha+\beta-1)}\right)\|x-y\| \text {. }
\end{aligned}
$$

since $R<1$, we conclude that $P_{2}$ is a contraction. Now, we show that $P_{1}$ is compact and continuous.
Continuity of $f$ implies that the operator $P_{1}$ is continuous. Also, $P_{1}$ is uniformly bounded on $B_{r^{\prime}}$ as

$$
\left\|P_{1} x\right\| \leq \frac{\|\theta\|_{L^{1}}}{\Gamma(\alpha+\beta+1)}
$$

Suppose that $0 \leq t_{1}<t_{2} \leq 1$. We have

$$
\begin{aligned}
\left|P_{1} x\left(t_{2}\right)-P_{1} x\left(t_{1}\right)\right| & \\
\leq & \frac{1}{\Gamma(\alpha+\beta)} \mid \int_{0}^{t_{1}}\left(\left(t_{2}-s\right)^{\alpha+\beta-1}-\left(t_{1}-s\right)^{\alpha+\beta-1}\right) \\
& \times f(s, x(s), \phi x(s), \varphi x(s)) d s+\int_{t_{1}}^{t_{2}}\left(t_{2}-s\right)^{\alpha+\beta-1} f \\
& \times(s, x(s), \phi x(s), \varphi x(s)) d s \mid \\
\leq & \frac{\|\theta\|_{L^{1}}}{\Gamma(\alpha+\beta+1)}\left[2\left(t_{2}-t_{1}\right)^{\alpha+\beta}+\left|t_{1}^{\alpha+\beta}-t_{2}^{\alpha+\beta}\right|\right] .
\end{aligned}
$$

Hence, $\left|P_{1} x\left(t_{2}\right)-P_{1} x\left(t_{1}\right)\right| \rightarrow 0$, as $t_{1} \rightarrow t_{2}$ independently from $x \in B_{r^{\prime}}$.

This shows that the operator $P_{1}$ is relatively compact on $B_{r^{\prime}}$. Hence, by the Arzela-Ascoli theorem, $P_{1}$ is compact on $B_{r^{\prime}}$.

Then, by Krasnoselskii's fixed point theorem, problem (1) has at least one solution on $B_{r^{\prime}}$.

\section{Example}

In this section, we give two examples to prove the applicability of our main results.

Example 9. Let us consider the following system:

$$
\left\{\begin{array}{l}
{ }^{c} D^{11 / 7}\left({ }^{c} D^{5 / 7}\right) x(t)=\frac{t^{2}}{200}\left(\frac{1}{1+|x(t)|}+\frac{1}{100} \int_{0}^{t} t^{4} s^{3} x(s) d s\right), \quad t \in[0,1], \\
x(0)=\frac{1}{500}\left(x\left(\frac{1}{19}\right)+x\left(\frac{4}{19}\right)+x\left(\frac{8}{19}\right)+x\left(\frac{11}{19}\right)\right), \quad x^{\prime}(0)=0, \\
\frac{1}{300} x(1)+\frac{1}{300} x^{\prime}(1)=\frac{1}{400}\left(\int_{2 / 19}^{3 / 19} x(s) d s+\int_{5 / 19}^{7 / 19} x(s) d s+\int_{9 / 19}^{10 / 19} x(s) d s\right) .
\end{array}\right.
$$

Here, $m=3, n=4, \beta=11 / 7, \alpha=5 / 7, a_{1}=a_{2}=a_{3}=a_{4}=$ $1 / 500, \mu_{1}=\mu_{2}=1 / 300, b_{1}=b_{2}=b_{3}=1 / 400, c_{1}=2 / 19, c_{2}=5$ $/ 19, c_{3}=9 / 19, d_{1}=3 / 19, d_{2}=7 / 19, d_{3}=10 / 19, f(t, x, y, z)=$ $\left(t^{2} / 200\right)(1 /(1+|x(t)|)+y(t)+z(t)), \quad \lambda(t, s)=\delta(t, s)=t^{4} s^{3} /$ 200 , and $\sigma(t)=t^{2} / 200$.

It follows that

$$
\lambda_{0}=\delta_{0}=\frac{1}{800}, \sigma^{*}=\frac{1}{600}, B_{1} \approx 1,3367, B_{2} \approx 117,95142, r_{1} \approx 0,0027
$$

By Theorem 7, we obtain that problem (30) has a unique solution. 
Example 10. Consider the following problem:

$$
\left\{\begin{array}{l}
{ }^{c} D^{18 / 11}\left({ }^{c} D^{6 / 11}\right) x(t)=\frac{t^{3}}{400}\left(\frac{|x(t)| e^{-t}}{1+|x(t)|}+\int_{0}^{t} \frac{(t+s)^{3}|x(s)|(\cos (s)+\sin (s))}{400(1+|x(s)|)}\right) t \in[0,1], \\
x(0)=\frac{1}{800}\left(x\left(\frac{12}{41}\right)+x\left(\frac{15}{41}\right)+x\left(\frac{18}{41}\right)+x\left(\frac{21}{41}\right)\right), \quad x^{\prime}(0)=0, \\
\frac{1}{600} x(1)+\frac{1}{600} x^{\prime}(1)=\frac{1}{700}\left(\int_{13 / 41}^{14 / 41} x(s) d s+\int_{16 / 41}^{17 / 41} x(s) d s+\int_{19 / 41}^{20 / 41} x(s) d s\right) .
\end{array}\right.
$$

Here, $m=3, n=4, \beta=18 / 11, \alpha=6 / 11, a_{1}=a_{2}=a_{3}=a_{4}$ $=1 / 800, \mu_{1}=\mu_{2}=1 / 600, b_{1}=b_{2}=b_{3}=1 / 700, c_{1}=13 / 41, c_{2}$ $=16 / 41, c_{3}=19 / 41, d_{1}=14 / 41, d_{2}=17 / 41, d_{3}=20 / 41, f(t$, $x, y, z)=\left(t^{3} / 400\right)\left(|x(t)| e^{-t} /(1+|x(t)|)+|y(t)| \cos (t) /(1+\mid y\right.$ $(t) \mid)+|z(t)| \sin (t) /(1+|z(t)|)), \quad \lambda(t, s)=\delta(t, s)=(t+s)^{3} /$ $400, \sigma(t)=t^{3} / 400$, and $\theta(t)=3 t^{3} / 400$.

It is clear that

$\lambda_{0}=\delta_{0}=\frac{15}{1600}, \sigma^{*}=\frac{1}{1600}, B_{1} \approx 1,3641, B_{2} \approx 229,3039, R \approx 0,000566$.

Then, problem (32) has at least one solution.

\section{Data Availability}

No data were used to support this study.

\section{Conflicts of Interest}

The authors declare that there are no conflicts of interest regarding the publication of this paper.

\section{References}

[1] V. Lakshmikantham, "Theory of fractional functional differential equations," Nonlinear Analysis: Theory, Methods \& Applications, vol. 69, no. 10, pp. 3337-3343, 2008.

[2] V. Lakshmikantham and A. S. Vatsala, "Basic theory of fractional differential equations," Nonlinear Analysis: Theory, Methods \& Applications, vol. 69, no. 8, pp. 2677-2682, 2008.

[3] R. Hilfer, Applications of Fractional Calculs in Physics, World Scientific, Singapore, 2000.

[4] K. S. Miller and B. Ross, An Introduction to the Fractional Calculus and Fractional Differential Equations, Wiley, New York, 1993.

[5] I. Podlubny, Fractional Differential Equations, Academic Press, New York, 1993.

[6] Y. Zhou, Basic Theory of Fractional Differential Equations, Xiangtan University, China, 2014.

[7] A. A. Kilbas, H. M. Srivastava, and J. J. Trujillo, Theory and applications of fractional differential equations, NorthHolland Mathematics Studies, vol. 204, Elsevier, Amsterdam, 2006.

[8] K. Hilal, L. Ibnelazyz, K. Guida, and S. Melliani, Existence of mild solutions for an impulsive fractional integro-differential equations with non-local condition, Springer Nature, Switzerland AG, 2019.

[9] K. Hilal, K. Guida, L. Ibnelazyz, and M. Oukessou, Existence results for an impulsive fractional integro-differential equations with non-compact semigroup, Springer Nature, Switzerland AG, 2019.

[10] K. Guida, K. Hilal, and L. Ibnelazyz, "Existence of mild solutions for a class of impulsive Hilfer fractional coupled systems," Advances in Mathematical Physics, vol. 2020, Article ID 8406509, 12 pages, 2020.

[11] M. A. Ragusa, "Commutators of fractional integral operators on vanishing-Morrey spaces," Journal of Global Optimization, vol. 40, no. 1-3, pp. 361-368, 2008.

[12] M. A. Ragusa and A. Tachikawa, "Boundary regularity of minimizers of $\mathrm{p}(\mathrm{x})$-energy functionals," Annales de l'Institut Henri Poincare (C) Non Linear Analysis, vol. 33, no. 2, pp. 451-476, 2016.

[13] E. Guariglia, "Riemann zeta fractional derivative - functional equation and link with primes," Advances in Difference Equations, vol. 2019, no. 1, 2019.

[14] E. Guariglia, "Fractional derivative of the Riemann zeta function," in Fractional Dynamics, C. Cattani, H. M. Srivastava, and X.-Y. Yang, Eds., pp. 357-368, De Gruyter, 2015.

[15] K. Hilal, L. Ibnelazyz, K. Guida, and S. Melliani, "Fractional Langevin equations with nonseparated integral boundary conditions," Advances in Mathematical Physics, vol. 2020, Article ID 3173764, 8 pages, 2020.

[16] M. Houas, "Existence results for a coupled system of fractional differential equations with multi-point boundary value problems," Mediterranean Journal of Modeling and Simulation, vol. 10, pp. 045-059, 2018.

[17] B. Li, S. Sun, Y. Li, and P. Zhao, "Multi-point boundary value problems for a class of Riemann-Liouville fractional differential equations," Advances in Difference Equations, vol. 2014, no. 1, Article ID 151, 2014.

[18] C. Li, X. Luo, and Y. Zhou, "Existence of positive solutions of the boundary value problem for nonlinear fractional differential equations," Computers \& Mathematcs with Applications, vol. 59, no. 3, pp. 1363-1375, 2010.

[19] J. Yang, J. C. Ma, S. Zhao, and Y. Ge, "Fractional multi-point boundary value problem of fractional differential equations," Mathematics in Practice and Theory, vol. 41, no. 11, pp. 188194, 2011.

[20] M. Rehman, R. Khan, and N. Asif, "Three point boundary value problems for nonlinear fractional differential equations," Acta Mathematica Scientia, vol. 31, no. 4, pp. 1337-1346, 2011.

[21] A. Cernea, "On a multi point boundary value problem for a fractional order differential inclusion," Arab Journal of Mathematical Sciences, vol. 19, no. 1, pp. 73-83, 2013.

[22] W. Jiang, B. Wang, and Z. Wang, "The existence of positive solutions for multi-point boundary value problems of fractional differential equations," Physics Procedia, vol. 25, pp. 958-964, 2012.

[23] B. Ahmed and S. Sivasundaram, "On four-point nonlocal boundary value problems of nonlinear integro-differential equations of fractional order," Applied Mathematics and Computation, vol. 217, no. 2, pp. 480-487, 2010.

[24] W. X. Zhou and Y. D. Chu, "Existence of solutions for fractional differential equations with multi-point boundary conditions," Communications in Nonlinear Science and Numerical Simulation, vol. 17, no. 3, pp. 1142-1148, 2012.

[25] D. Baleanu, S. Z. Nazeni, and S. Rezapour, "Existence and uniqueness of solutions for multi-term nonlinear fractional integro-differential equations," Advances in Difference Equations, vol. 2013, no. 1, 2013. 
[26] A. Bashir, S. Ntouyas, R. Agrwal, and A. Alsaedi, "Existence results for sequential fractional integro-differential equations with nonlocal multi-point and strip conditions," Boundary Value Problems, vol. 2016, 16 pages, 2016.

[27] A. Bragdi, A. Frioui, and A. G. Lakoud, "Existence of solutions for nonlinear fractional integro-differential equations," Advances in Difference Equations, vol. 2020, 9 pages, 2020.

[28] A. Krasnoselskii, "Two remarks on the method of successive approximations," Uspekhi Matematicheskikh Nauk, vol. 10, pp. 123-127, 1955. 